\title{
Mass spectrometry-based proteome profiling of extracellular vesicles and their roles in cancer biology
}

\author{
Raju Bandu', Jae Won $\mathrm{Oh}^{1}$ and Kwang Pyo Kim (i) ${ }^{1,2}$
}

\begin{abstract}
Over the past three decades, extracellular vesicles (EVs) have arisen as important mediators of intercellular communication that are involved in the transmission of biological signals between cells to regulate various biological processes. EVs are largely responsible for intercellular communication through the delivery of bioactive molecules, such as proteins, messenger RNAs (mRNAs), microRNAs (miRNAs), DNAs, lipids, and metabolites. EVs released from cancer cells play a significant role in signal transduction between cancer cells and the surrounding cells, which contributes to the formation of tumors and metastasis in the tumor microenvironment. In addition, EVs released from cancer cells migrate to blood vessels and flow into various biological fluids, including blood and urine. EVs and EVloaded functional cargoes, including proteins and miRNAs, found in these biological fluids are important biomarkers for cancer diagnosis. Therefore, EV proteomics greatly contributes to the understanding of carcinogenesis and tumor progression and is critical for the development of biomarkers for the early diagnosis of cancer. To explore the potential use of EVs as a gateway to understanding cancer biology and to develop cancer biomarkers, we discuss the mass spectrometric identification and characterization of EV proteins from different cancers. Information provided in this review may help in understanding recent progress regarding EV biology and the potential roles of EVs as new noninvasive biomarkers and therapeutic targets.
\end{abstract}

\section{Introduction}

Extracellular vesicles (EVs) are membrane-surrounded vesicles released by numerous cell types into the extracellular microenvironment ${ }^{1-3}$. EVs are involved in cell-cell communication, coagulation, inflammation, immune response modulation, and disease progression ${ }^{2,4-7}$. Although EVs vary in size, biological function, and components, their significance in cancer progression and the potential use of EV molecules as novel cancer biomarkers has gradually increased. Cancer cells actively release EVs into neighboring tissues, and these EVs play

Correspondence: Kwang Pyo Kim (kimkp@khu.ac.kr)

'Department of Applied Chemistry, Institute of Natural Science, Global Center for Pharmaceutical Ingredient Materials, Kyung Hee University, Yongin,

Republic of Korea

2Department of Biomedical Science and Technology, Kyung Hee Medical

Science Research Institute, Kyung Hee University, Seoul, Republic of Korea dynamic roles in cancer progression and metastasis, invasion, angiogenesis, tumorigenesis, and immune modulation $^{8-10}$. EVs released by cancer cells are usually chosen as a gateway in the search for biomarkers for a specific cancer type. Recent results pertaining to EV-cargo molecules, including proteins and miRNAs, are summarized in EVpedia (http://evpedia.info), an integrated and comprehensive database of EVs ${ }^{11}$.

The main focus of this review is proteome profiling of EVs using mass spectrometry (MS)-based proteomic approaches. We discuss the mass spectral characterization of isolated EV proteins from different cancers and the use of these proteins as predictive cancer biomarkers. Additionally, we summarize the key characteristics of enriched proteins in cancer-associated EVs as potential therapeutic targets and provide novel information on their roles in cancer development and progression. Information

\section{(c) The Author(s) 2019}

(c) (i) Open Access This article is licensed under a Creative Commons Attribution 4.0 International License, which permits use, sharing, adaptation, distribution and reproduction c. in any medium or format, as long as you give appropriate credit to the original author(s) and the source, provide a link to the Creative Commons license, and indicate if changes were made. The images or other third party material in this article are included in the article's Creative Commons license, unless indicated otherwise in a credit line to the material. If material is not included in the article's Creative Commons license and your intended use is not permitted by statutory regulation or exceeds the permitted use, you will need to obtain permission directly from the copyright holder. To view a copy of this license, visit http://creativecommons.org/licenses/by/4.0/. 
provided in this review may help in understanding recent progress regarding EV biology and the prospective roles of EVs as new noninvasive biomarkers and therapeutic targets, as well as emerging therapeutic opportunities and associated challenges.

\section{Classification of EVs}

EVs are small spherical vesicles that are secreted into the extracellular milieu by many cell types. The term "EV" was invented by the International Society of Extracellular Vesicles (ISEV) and is used to define all phospholipid bilayer-bound vesicles that are secreted by cells into the extracellular microenvironment, regardless of the differences in biogenesis, size, and composition ${ }^{12,13}$. The roles of EVs in different physiological and pathological processes have made them a novel field of research. EVs are categorized into several subtypes based on their size, density, shape, subcellular origin, function, and molecular cargo $^{14}$. The four major subtypes of EVs are exosomes, microvesicles, apoptotic bodies, and oncosomes (Table 1 and Fig. 1). Exosomes are 30-200-nm-sized homogeneous membrane vesicles, and they form through the endosomal trafficking pathway ${ }^{5,15,16}$. Exosomes contain late endosomal markers, even though biochemically indistinguishable vesicles can bud directly from the plasma membrane ${ }^{16,17}$. They play critical roles in cell-cell communications, such as that occurring during the regulation of cell and tissue homeostasis, as well as in pathological conditions ${ }^{18}$. Microvesicles are 100-1000-nm-sized heterogeneous membrane vesicles that originate via outward budding and the fission of the plasma membrane due to dynamic interactions during phospholipid redistribution. Phospholipid distribution is controlled by aminophospholipid translocases ${ }^{16,18-24}$ and cytoskeletal protein contraction. Microvesicles are released mostly under cellular stress or in pathological processes ${ }^{18}$. Like exosomes, microvesicles transfer bioactive molecules into target cells. Apoptotic bodies $(>1 \mu \mathrm{m})$ are released by cells that undergo the apoptosis process or programmed cell death ${ }^{18,24}$, and they can be characterized by cellular organelles and DNA. Finally, the vesicles named "oncosomes" are much larger than most other EV types characterized to date $(1-10 \mu \mathrm{m})$. Owing to their unusual size, large oncosomes might have unique properties in vivo and would provide novel opportunities for tumor profiling ${ }^{25}$.

EVs contain proteins, lipids, metabolites, and RNAs. However, the mechanisms by which these components enter EVs remain obscure. EVs are shed from almost all cell types and are present in biological fluids and conditioned cell culture media. EVs are involved in cell-cell communication, coagulation, inflammation, immune response modulation, and disease progression ${ }^{4-7}$. The functional roles of EVs in intercellular communication have made them of major interest in many scientific fields. 


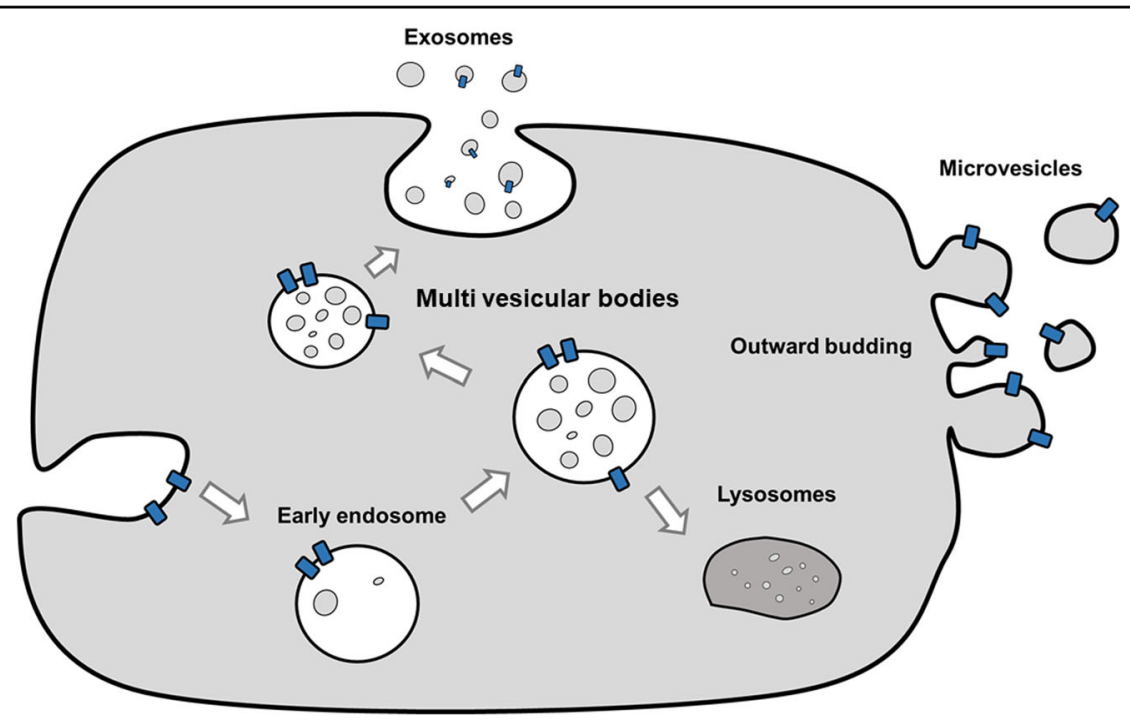

Fig. 1 Biogenesis of four major subtypes of extracellular vesicles

The biomolecular composition of EVs could play a significant role in disease progression in several neurodegenerative diseases as well as in cancer.

\section{MS in EV proteome analyses}

EV proteome analysis is a novel approach and is part of the growing interest in proteomics cancer research. Over the past three decades, many proteomics studies performed on EVs have elucidated their diverse roles. Largescale proteomics datasets and protein-interaction networks have established significant relationships between EV proteins, which improves the understanding of vesicle biogenesis and pathophysiological roles ${ }^{24,26,27}$. Proteomic studies on EVs from different origins have also suggested a controlled protein-sorting mechanism and the random packaging of EV proteins from various cell types that contain common vesicular proteins. Furthermore, proteomic studies of EVs have produced a highthroughput vesicular proteome dataset from various cell types and body fluids ${ }^{28}$. Since EVs are normally isolated in small amounts, better sensitivity is required for their analysis. Liquid chromatography (nanoscale or ultra-high performance)-electrospray ionization tandem mass spectrometry (LC/ESI-MS/MS) is the most popular and versatile analytical technique to study the molecular contents of EVs. In particular, nano-ESI-MS/MS provides high sensitivity and resolution, allowing the detection, identification, characterization, and quantification of thousands of proteins from even a single EV sample. Similar to other biological fields, LC-MS/MS-based technological platforms have become the most popular fundamental tools for elucidating the structural and functional architecture of EVs. The fragment ions from ESI (positive- and negative-ion) tandem MS experiments provide the composition, unambiguous structural characterization, and proper identification of proteins present in various biological samples. Due to the high sensitivity and small initial sample volumes required for MS, MS-based proteomic analysis has increased the understanding of EV protein content. Several investigators $^{26,29-32}$ have used ESI tandem MS experiments in combination with chromatographic methods (HPLC, UHPLC, UPLC, and nano LC) to profile and structurally characterize proteins in various cancer cells, tissues, biofluids, and biological samples, which have been summarized in Table 2.

\section{EV proteomes in various cancers and biomarker discovery}

Proteomic analysis of EVs has revealed significant changes in protein expression under various physiological and pathological conditions ${ }^{26,29,30}$. Characterization of these proteomic profiles may be useful in understanding disease pathogenesis and assisting in the discovery of new biomarkers for different diseases. The secretion of EVs from several types of tumor cells is a significant means of conditioning and altering the tumor microenvironment by malignant cells ${ }^{31,32}$. Multiple studies have reported that the secretion of EVs from cancer cells contributes to angiogenesis, metastasis, tumor formation, and disease progression $^{2,10,31,32}$. EVs are more attractive sources of biomarkers because of their biological consequences and relatively noninvasive accessibility in a wide range of biological fluids. EVs have been studied in relation to numerous cancers, such as colorectal ${ }^{27,33}$, bladder $^{34}$, prostate $^{35}$, pancreatic ${ }^{36}$, breast ${ }^{37}$, gastric ${ }^{38}$, lung ${ }^{39}$, blood $^{40}$, ovarian ${ }^{41}$, cholangiocarcinoma ${ }^{42}$, hepatocellular carcinoma $^{43}$, and oral squamous cell carcinoma ${ }^{44}$ 


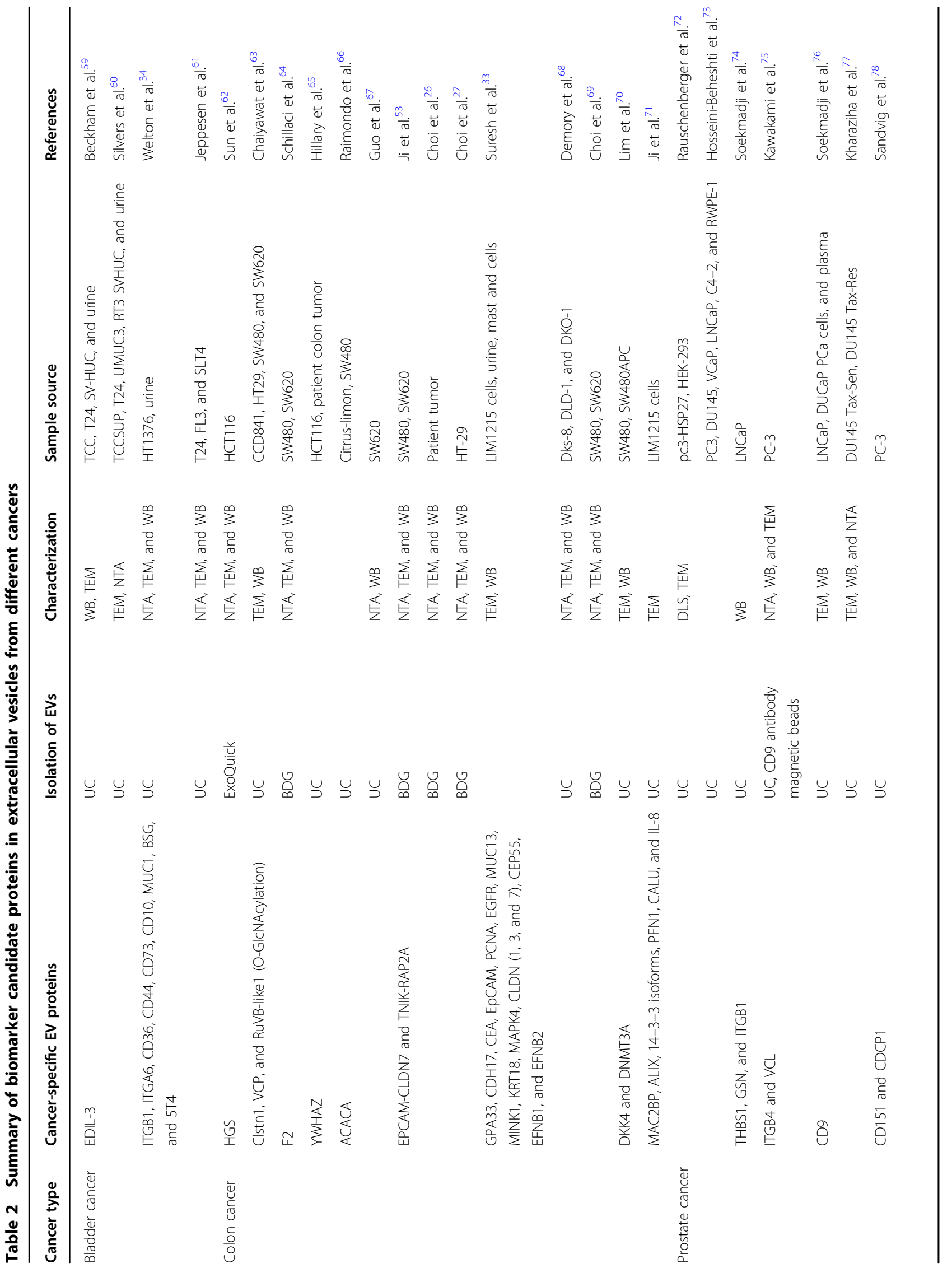




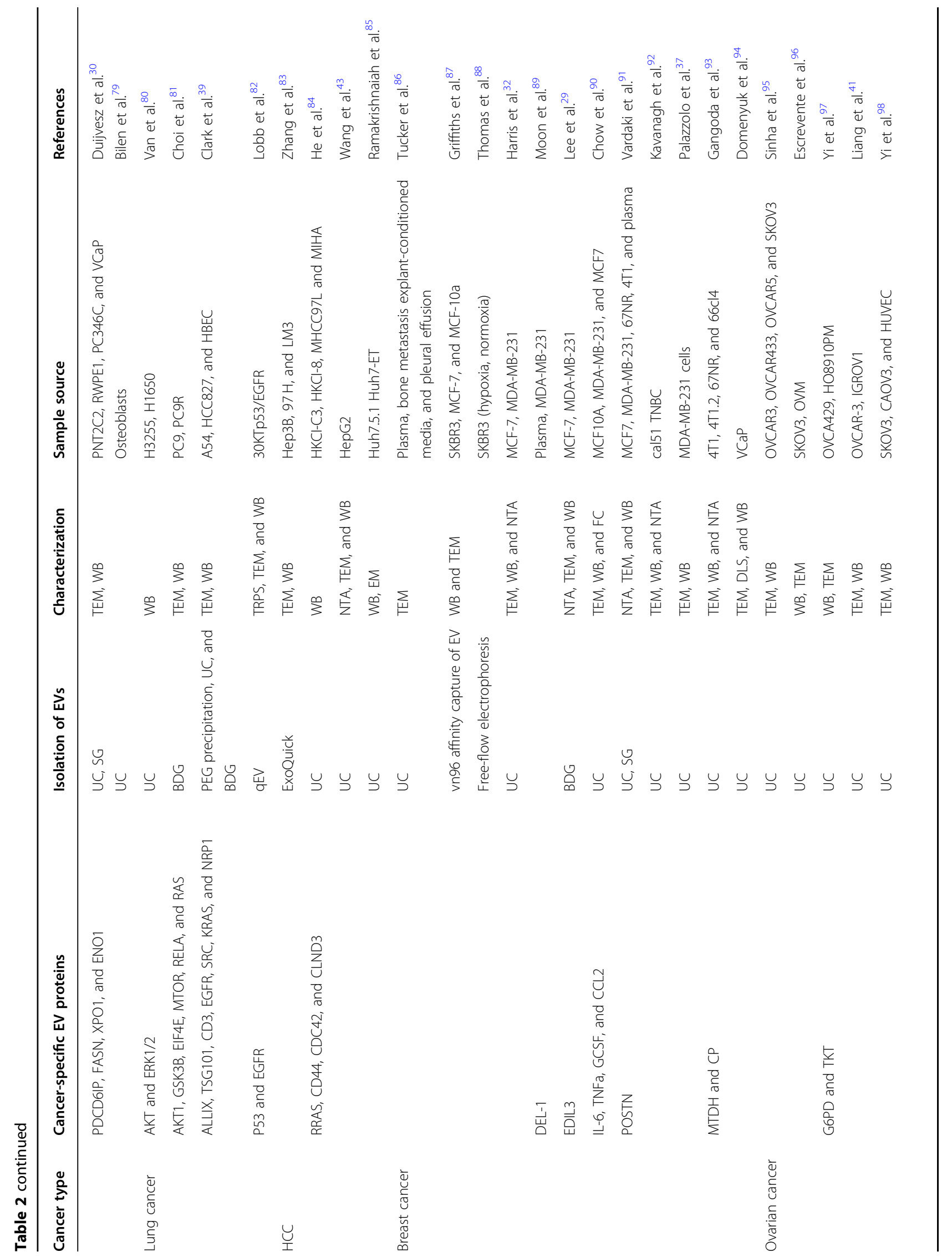




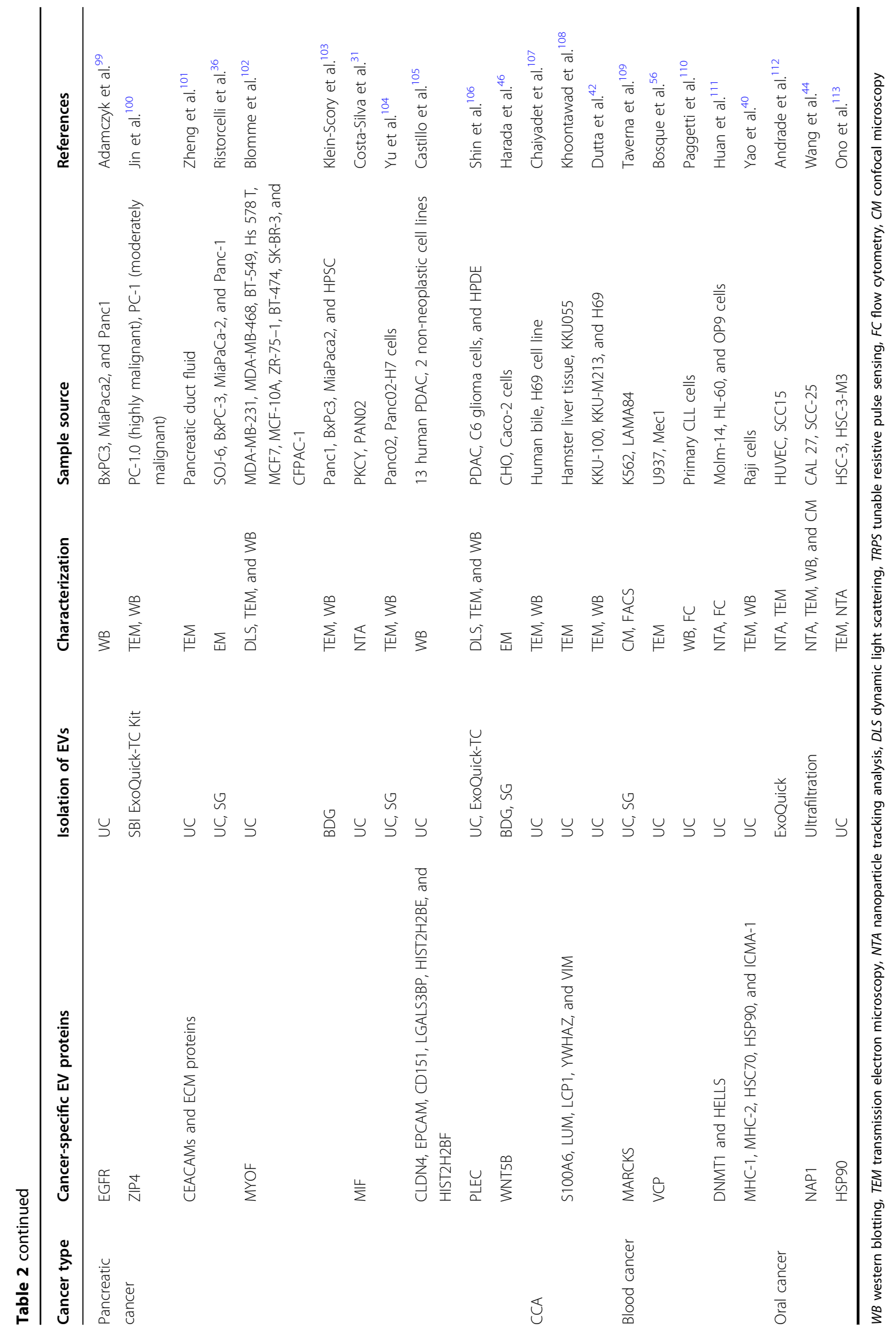




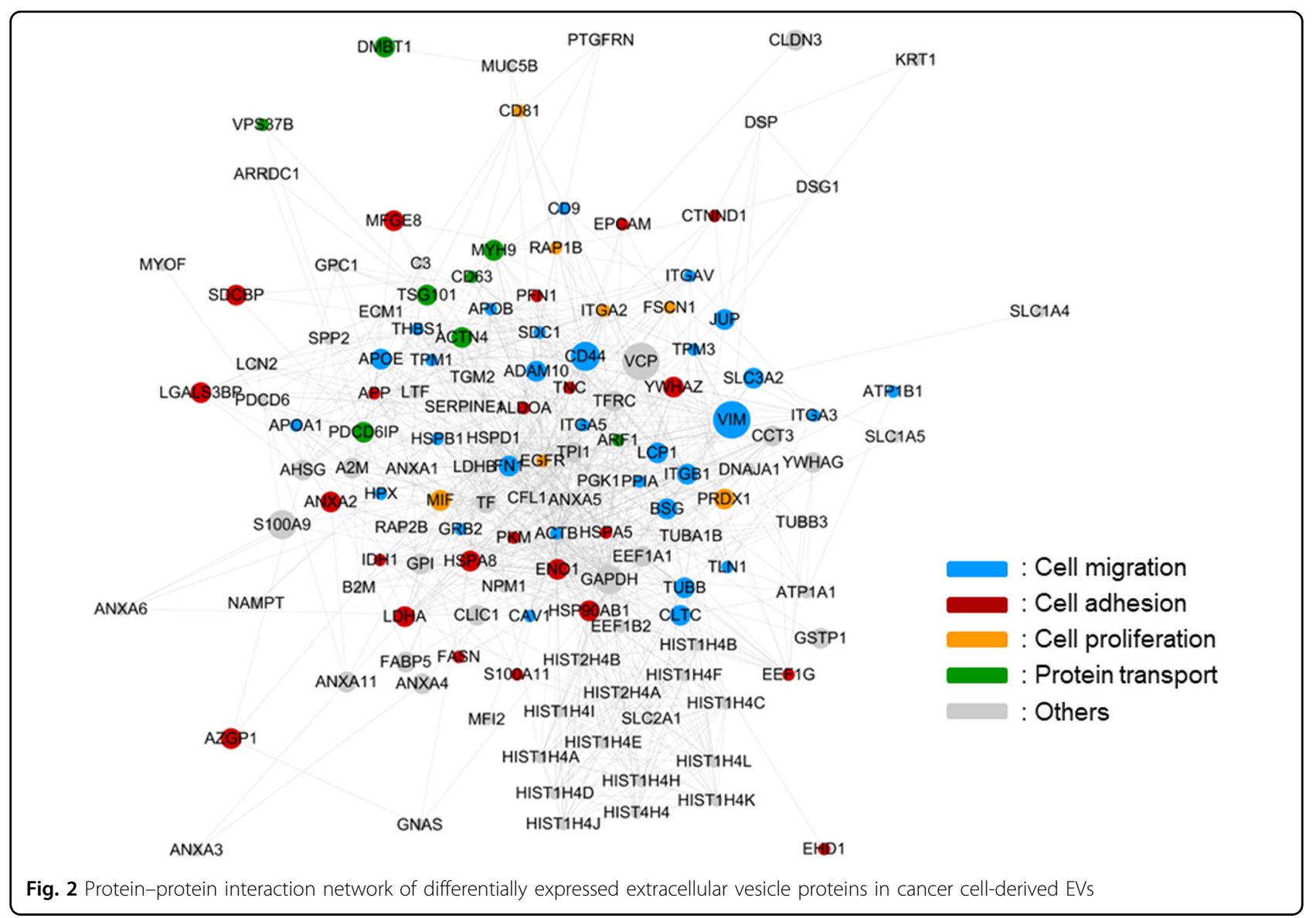

(Table 2), as well as cardiovascular diseases ${ }^{45}$ and malignancies of the central nervous system ${ }^{21}$. The proteomic analysis of EVs, specifically the analysis of their protein composition, may be helpful for further understanding the mechanisms of their biogenesis and their functional roles. Molecular communication between cancer cells and their stromal microenvironment is a key factor for cancer progression $^{46,47}$. In conjunction with typical secretory pathways, it was proposed that these small membranous vesicles are alternate mediators of intercellular communication ${ }^{19}$. EVs carry an effector-rich proteome with the ability to control different functional properties of the recipient $\mathrm{cell}^{48}$. The protein composition of EVs from different sources was studied previously by using $\mathrm{MS}^{30,49-54}$, providing a robust basis for the identification of biomarker proteins in EVs for the purpose of quality control research. A thorough understanding of the protein composition of EV subtypes and the extent to which EV composition reflects the source cell composition is essential for further development of diagnostics and therapeutics. Although EVs are secreted by almost all cell types, some available data suggest the enhanced release of EVs under pathological conditions, such as cancer ${ }^{55}$. It is reasonable to expect that these vesicles may also play key roles in tumorigenesis since they can facilitate distant intercellular communication. Tumor-derived EVs typically carry tumor antigens, and functional proteins can be transferred to recipient cells through $\mathrm{EVs}^{23,54,56}$. A better understanding of the molecular bases underlying cancer invasion and metastasis is necessary to develop effective targets for therapy.

EV proteins from many cancers have similar biological processes and functions. To understand the functions of differentially expressed proteins (DEPs) in cancer, we performed gene ontology analysis on a variety of DEPs ${ }^{57}$. As expected, the EV-DEPs from different cancer types were implicated in similar biological processes, such as cell adhesion, migration, and transport. Considering that EVs are potential metastasis factors, those proteins appear to be relevant for cancer metastasis or cancer cell proliferation. Of the 12 different cancers evaluated, we observed that DEPs that overlapped more than five times were primarily related to cancer metastasis or cancer cell proliferation, and many of the DEPs had strong interactions with each other (Fig. 2). Even though the selection of these DEPs from different cancers was biased, the roles of EVs in different cancers focused mainly on cell adhesion and cell migration. 


\section{Conclusions}

In this review, we summarized different EV studies to discuss the potential of EVs in cancer treatment. All studies discussed in this review indicated that the specific protein composition of various EVs has high potential for identifying different cancers. The majority of these studies revealed the relationship of cancer with changes in the protein contents of various body fluids. Moreover, we have highlighted the emerging roles of EVs in cancer, specifically their role in metastasis, which opens the possibility of the rapid translation of EV research for clinical applications in diagnosis, prognosis, and treatment. Ultimately, the majority of the investigations discussed in this review need further verification in large-cohort, multicenter clinical studies. In the future, highly reliable EV proteome data could be combined with well-developed current popular genomic and other "omics-" studies to provide extended knowledge of EVs from the perspective of systems biology approaches.

\section{Future perspectives}

There are many perspectives on the potential contribution of EV research for the development of cancer therapeutics and diagnosis. EVs could play key roles in intercellular communication during cancer development, which may offer new therapeutic strategies for various cancers. EV protein composition in different body fluids reveals the overall condition of the patient and is also useful for screening the efficacy and toxicity of anticancer treatments. Additionally, EVs could be used as cancer vaccines and drug delivery components. Moreover, the inhibition of intercellular communication through EVs might provide opportunities to suppress tumor progression. In the near future, clinical applications of EVs could contribute to cancer management and treatment. However, before EV-targeted therapy can be applied in cancer, the identification of cancerspecific genes or molecules that are crucial for EV communication is necessary.

\section{Acknowledgements}

This work was supported by a grant from Kyung Hee University in 2017 (KHU20171191) and the GRRC program of Gyeonggi province [GRRC-kyunghee2018 (B03)].

\section{Conflict of interest}

The authors declare that they have no conflict of interest.

\section{Publisher's note}

Springer Nature remains neutral with regard to jurisdictional claims in published maps and institutional affiliations.

Received: 7 November 2018 Accepted: 12 December 2018. Published online: 15 March 2019

\section{References}

1. Perez-Hernandez, D. et al. The intracellular interactome of tetraspaninenriched microdomains reveals their function as sorting machineries toward exosomes. J. Biol. Chem. 288, 11649-11661 (2013).

2. Kahlert, C. \& Kalluri, R. Exosomes in tumor microenvironment influence cancer progression and metastasis. J. Mol. Med. 91, 431-437 (2013).

3. Raposo, G. \& Stoorvogel, W. Extracellular vesicles: exosomes, microvesicles, and friends. J. Cell. Biol. 200, 373-383 (2013).

4. Mathivanan, S., Ji, H. \& Simpson, R. J. Exosomes: extracellular organelles important in intercellular communication. J. Proteom. 73, 1907-1920 (2010).

5. Thery, C., Ostrowski, M. \& Segura, E. Membrane vesicles as conveyors of immune responses. Nat. Rev. Immunol. 9, 581-593 (2009).

6. Gabrilovich, D. I., Ostrand-Rosenberg, S. \& Bronte, V. Coordinated regulation of myeloid cells by tumours. Nat. Rev. Immunol. 12, 253-268 (2012).

7. Del Conde, I., Shrimpton, C. N., Thiagarajan, P. \& Lopez, J. A. Tissue-factorbearing microvesicles arise from lipid rafts and fuse with activated platelets to initiate coagulation. Blood 106, 1604-1611 (2005).

8. Andaloussi, E. L., Mager, S., Breakefield, I., X. O. \& Wood, M. J. Extracellular vesicles: biology and emerging therapeutic opportunities. Nat. Rev. Drug. Discov. 12, 347-357 (2013)

9. Valenti, R. et al. Human tumor-released microvesicles promote the differentiation of myeloid cells with transforming growth factor-beta-mediated suppressive activity on T lymphocytes. Cancer Res. 66, 9290-9298 (2006).

10. Kim, C. W. et al. Extracellular membrane vesicles from tumor cells promote angiogenesis via sphingomyelin. Cancer Res. 62, 6312-6317 (2002).

11. Kim, D. K. et al. EVpedia: a community web portal for extracellular vesicles research. Bioinformatics 31, 933-939 (2015).

12. Thery, C., Zitvogel, L. \& Amigorena, S. Exosomes: composition, biogenesis and function. Nat. Rev. Immunol. 2, 569-579 (2002).

13. O'Driscoll, L. Expanding on exosomes and ectosomes in cancer. N. Engl. J. Med. 372, 2359-2362 (2015).

14. Jang, S. C. et al. In vivo kinetic biodistribution of nano-sized outer membrane vesicles derived from bacteria. Small 11, 456-461 (2015).

15. Gould, S. J. \& Raposo, G. As we wait: coping with an imperfect nomenclature for extracellular vesicles. J. Extracell Vesicles 2, https://doi.org/10.3402/jev. v2i0.20389 (2013).

16. Bobrie, A., Colombo, M., Krumeich, S., Raposo, G. \& Thery, C. Diverse subpopulations of vesicles secreted by different intracellular mechanisms are present in exosome preparations obtained by differential ultracentrifugation. J. Extracell Vesicles 1, https://doi.org/10.3402/jev.v1i0.18397 (2012).

17. Kowal, J., Tkach, M. \& Thery, C. Biogenesis and secretion of exosomes. Curr. Opin. Cell Biol. 29, 116-125 (2014).

18. Booth, A. M. et al. Exosomes and HIV Gag bud from endosome-like domains of the T cell plasma membrane. J. Cell. Biol. 172, 923-935 (2006).

19. Akers, J. C., Gonda, D., Kim, R., Carter, B. S. \& Chen, C. C. Biogenesis of extracellular vesicles (EV): exosomes, microvesicles, retrovirus-like vesicles, and apoptotic bodies. J. Neuro-Oncol. 113, 1-11 (2013).

20. Colombo, M., Raposo, G. \& Thery, C. Biogenesis, secretion, and intercellular interactions of exosomes and other extracellular vesicles. Annu. Rev. Cell. Dev. Biol. 30, 255-289 (2014).

21. Skog, J. et al. Glioblastoma microvesicles transport RNA and proteins that promote tumour growth and provide diagnostic biomarkers. Nat. Cell Biol. 10, 1470-1476 (2008).

22. Gyorgy, B. et al. Membrane vesicles, current state-of-the-art: emerging role of extracellular vesicles. Cell. Mol. Life Sci. 68, 2667-2688 (2011).

23. Baj-Krzyworzeka, M., Szatanek, R., Weglarczyk, K., Baran, J. \& Zembala, M. Tumour-derived microvesicles modulate biological activity of human monocytes. Immunol. Lett. 113, 76-82 (2007).

24. Choi, D. S., Kim, D. K., Kim, Y. K. \& Gho, Y. S. Proteomics, transcriptomics and lipidomics of exosomes and ectosomes. Proteomics 13, 1554-1571 (2013).

25. Minciacchi, V. R., Freeman, M. R. \& Di Vizio, D. Extracellular vesicles in cancer: exosomes, microvesicles and the emerging role of large oncosomes. Semin. Cell. Dev. Biol. 40, 41-51 (2015).

26. Choi, D. S. et al. Proteomic analysis of microvesicles derived from human colorectal cancer ascites. Proteomics 11, 2745-2751 (2011).

27. Choi, D. S. et al. Proteomic analysis of microvesicles derived from human colorectal cancer cells. J. Proteome Res. 6, 4646-4655 (2007).

28. Raimondo, F., Morosi, L., Chinello, C., Magni, F. \& Pitto, M. Advances in membranous vesicle and exosome proteomics improving biological understanding and biomarker discovery. Proteomics 11, 709-720 (2011). 
29. Lee, J. E. et al. Identification of EDIL3 on extracellular vesicles involved in breast cancer cell invasion. J. Proteom. 131, 17-28 (2016).

30. Duijvesz, D. et al. Proteomic profiling of exosomes leads to the identification of novel biomarkers for prostate cancer. PLOS ONE 8, e82589 (2013).

31. Costa-Silva, B. et al. Pancreatic cancer exosomes initiate pre-metastatic niche formation in the liver. Nat. Cell Biol. 17, 816-826 (2015).

32. Harris, D. A. et al. Exosomes released from breast cancer carcinomas stimulate cell movement. PLoS ONE 10, e0117495 (2015).

33. Mathivanan, S. et al. Proteomics analysis of A33 immunoaffinity-purified exosomes released from the human colon tumor cell line LIM1215 reveals a tissue-specific protein signature. Mol. Cell. Proteom. 9, 197-208 (2010).

34. Welton, J. L. et al. Proteomics analysis of bladder cancer exosomes. Mol. Cell. Proteom. 9, 1324-1338 (2010).

35. Jansen, F. H. et al. Exosomal secretion of cytoplasmic prostate cancer xenograft-derived proteins. Mol. Cell. Proteom. 8, 1192-1205 (2009).

36. Ristorcelli, E. et al. Human tumor nanoparticles induce apoptosis of pancreatic cancer cells. FASEB J. 22, 3358-3369 (2008).

37. Palazzolo, G. et al. Proteomic analysis of exosome-like vesicles derived from breast cancer cells. Anticancer Res. 32, 847-860 (2012).

38. $\mathrm{Fu}, \mathrm{H}$. et al. Exosomal TRIM 3 is a novel marker and therapy target for gastric cancer. J. Exp. Clin. Cancer Res. 37, 162 (2018).

39. Clark, D. J., Fondrie, W. E., Yang, A. \& Mao, L. Triple SILAC quantitative proteomic analysis reveals differential abundance of cell signaling proteins between normal and lung cancer-derived exosomes. J. Proteom. 133 161-169 (2016).

40. Yao, Y. et al. Proteomic analysis of exosomes derived from human lymphoma cells. Eur. J. Med. Res. 20, 8 (2015).

41. Liang, B. et al. Characterization and proteomic analysis of ovarian cancerderived exosomes. J. Proteom. 80, 171-182 (2013).

42. Dutta, S. et al. Proteomics profiling of cholangiocarcinoma exosomes: a potential role of oncogenic protein transferring in cancer progression. Biochim. Biophys. Acta 1852, 1989-1999 (2015).

43. Wang, S. et al. Exosomes released by hepatocarcinoma cells endow adipocytes with tumor-promoting properties. J. Hematol. Oncol. 11, 82 (2018).

44. Wang, Y. et al. Oral cancer-derived exosomal NAP1 enhances cytotoxicity of natural killer cells via the IRF-3 pathway. Oral. Oncol. 76, 34-41 (2018).

45. Zhang, Y. et al. Secreted monocytic miR-150 enhances targeted endothelial cell migration. Mol. Cell 39, 133-144 (2010).

46. Harada, T. et al. Wnt5b-associated exosomes promote cancer cell migration and proliferation. Cancer Sci. 108, 42-52 (2017).

47. Kharmate, G., Hosseini-Beheshti, E., Caradec, J., Chin, M. Y. \& Tomlinson Guns, E. S. Epidermal growth factor receptor in prostate cancer derived exosomes. PLOS ONE 11, e0154967 (2016).

48. Keller, S., Sanderson, M. P., Stoeck, A. \& Altevogt, P. Exosomes: from biogenesis and secretion to biological function. Immunol. Lett. 107, 102-108 (2006).

49. Kreimer, S. et al. Mass-spectrometry-based molecular characterization of extracellular vesicles: lipidomics and proteomics. J. Proteome Res. 14, 2367-2384 (2015).

50. Clark, D. J. et al. Redefining the breast cancer exosome proteome by tandem mass tag quantitative proteomics and multivariate cluster analysis. Anal. Chem. 87, 10462-10469 (2015).

51. Wang, Z., Hill, S., Luther, J. M., Hachey, D. L. \& Schey, K. L. Proteomic analysis of urine exosomes by multidimensional protein identification technology (MudPIT). Proteomics 12, 329-338 (2012).

52. Kalra, H. et al. Comparative proteomics evaluation of plasma exosome isolation techniques and assessment of the stability of exosomes in normal human blood plasma. Proteomics 13, 3354-3364 (2013).

53. Ji, $\mathrm{H}$. et al. Proteome profiling of exosomes derived from human primary and metastatic colorectal cancer cells reveal differential expression of key metastatic factors and signal transduction components. Proteomics 13, 1672-1686 (2013).

54. Graner, M. W. et al. Proteomic and immunologic analyses of brain tumor exosomes. FASEB J. 23, 1541-1557 (2009).

55. Ohno, S., Ishikawa, A. \& Kuroda, M. Roles of exosomes and microvesicles in disease pathogenesis. Adv. Drug Deliv. Rev. 65, 398-401 (2013).

56. Bosque, A. et al. Comparative proteomics of exosomes secreted by tumoral Jurkat T cells and normal human T cell blasts unravels a potential tumorigenic role for valosin-containing protein. Oncotarget 7, 29287-29305 (2016).
57. Huang da, W., Sherman, B. T. \& Lempicki, R. A. Systematic and integrative analysis of large gene lists using DAVID bioinformatics resources. Nat. Protoc. 4, 44-57 (2009).

58. Barteneva, N. S. et al. Circulating microparticles: square the circle. Bmc. Cell. Biol. 14, 23 (2013).

59. Beckham, C. J. et al. Bladder cancer exosomes contain EDIL-3/Del1 and facilitate cancer progression. J. Urol. 192, 583-592 (2014).

60. Silvers, C. R., Miyamoto, H., Messing, E. M., Netto, G. J. \& Lee, Y. F. Characterization of urinary extracellular vesicle proteins in muscle-invasive bladder cancer. Oncotarget 8, 91199-91208 (2017).

61. Jeppesen, D. K. et al. Quantitative proteomics of fractionated membrane and lumen exosome proteins from isogenic metastatic and nonmetastatic bladder cancer cells reveal differential expression of EMT factors. Proteomics 14, 699-712 (2014).

62. Sun, Y. et al. A novel TP53 pathway influences the HGS-mediated exosome formation in colorectal cancer. Sci. Rep. 6, 28083 (2016).

63. Chaiyawat, P. et al. Elevated O-GlcNAcylation of extracellular vesicle proteins derived from metastatic colorectal cancer cells. Cancer Genom. Proteom. 13 387-398 (2016).

64. Schillaci, O. et al. Exosomes from metastatic cancer cells transfer amoeboid phenotype to non-metastatic cells and increase endothelial permeability: their emerging role in tumor heterogeneity. Sci. Rep. 7, 4711 (2017).

65. Mulvey, H. E. et al. Extracellular vesicle-mediated phenotype switching in malignant and non-malignant colon cells. Bmc. Cancer 15, 571 (2015).

66. Raimondo, S. et al. Label-free quantitative proteomic profiling of colon cancer cells identifies acetyl-CoA carboxylase alpha as antitumor target of Citrus limon-derived nanovesicles. J. Proteom. 173, 1-11 (2018).

67. Guo, J. et al. Phosphoproteome characterization of human colorectal cancer SW620 cell-derived exosomes and new phosphosite discovery for C-HPP. J. Proteome Res. 15, 4060-4072 (2016).

68. Demory Beckler, M. et al. Proteomic analysis of exosomes from mutant KRAS colon cancer cells identifies intercellular transfer of mutant KRAS. Mol. Cell. Proteom. 12, 343-355 (2013).

69. Choi, D. S. et al. Quantitative proteomics of extracellular vesicles derived from human primary and metastatic colorectal cancer cells. J. Extracell Vesicles 1, https://doi.org/10.3402/jev.v1i0.18704 (2012).

70. Lim, J. W. et al. Restoration of full-length APC protein in SW480 colon cancer cells induces exosome-mediated secretion of DKK-4. Electrophoresis 33, 1873-1880 (2012).

71. Ji, H., Greening, D. W., Kapp, E. A., Moritz, R. L. \& Simpson, R. J. Secretomebased proteomics reveals sulindac-modulated proteins released from colon cancer cells. Proteom. Clin. Appl. 3, 433-451 (2009).

72. Rauschenberger, L. et al. Exosomal particles secreted by prostate cancer cells are potent mRNA and protein vehicles for the interference of tumor and tumor environment. Prostate 76, 409-424 (2016).

73. Hosseini-Beheshti, E., Pham, S., Adomat, H., Li, N. \& Tomlinson Guns, E. S. Exosomes as biomarker enriched microvesicles: characterization of exosomal proteins derived from a panel of prostate cell lines with distinct AR phenotypes. Mol. Cell. Proteom. 11, 863-885 (2012).

74. Soekmadji, C., Rockstroh, A., Ramm, G. A., Nelson, C. C. \& Russell, P. J. Extracellular vesicles in the adaptive process of prostate cancer during inhibition of androgen receptor signaling by Enzalutamide. Proteomics 17, https://doi. org/10.1002/pmic.201600427 (2017).

75. Kawakami, K. et al. Integrin beta4 and vinculin contained in exosomes are potential markers for progression of prostate cancer associated with taxaneresistance. Int. J. Oncol. 47, 384-390 (2015).

76. Soekmadji, C. et al. Modulation of paracrine signaling by CD9 positive small extracellular vesicles mediates cellular growth of androgen deprived prostate cancer. Oncotarget 8, 52237-52255 (2017).

77. Kharaziha, P. et al. Molecular profiling of prostate cancer derived exosomes may reveal a predictive signature for response to docetaxel. Oncotarget $\mathbf{6}$ 21740-21754 (2015)

78. Sandvig, K. \& Llorente, A. Proteomic analysis of microvesicles released by the human prostate cancer cell line PC-3. Mol. Cell. Proteom. 11, M111-012914 (2012)

79. Bilen, M. A. et al. Proteomics profiling of exosomes from primary mouse osteoblasts under proliferation versus mineralization conditions and characterization of their uptake into prostate cancer cells. J. Proteome Res. 16, 2709-2728 (2017). 
80. van der Mijn, J. C. et al. Analysis of AKT and ERK1/2 protein kinases in extracellular vesicles isolated from blood of patients with cancer. J. Extracell. Vesicles 3, 25657 (2014)

81. Choi, D. Y. et al. Extracellular vesicles shed from gefitinib-resistant nonsmal cell lung cancer regulate the tumor microenvironment. Proteomics $\mathbf{1 4}$, 1845-1856 (2014).

82. Lobb, R. J. et al. Oncogenic transformation of lung cells results in distinct exosome protein profile similar to the cell of origin. Proteomics 17, https:// doi.org/10.1002/pmic.201600432 (2017).

83. Zhang, J. et al. Motile hepatocellular carcinoma cells preferentially secret sugar metabolism regulatory proteins via exosomes. Proteomics 17, https:// doi.org/10.1002/pmic.201700103 (2017).

84. He, M. et al. Hepatocellular carcinoma-derived exosomes promote motility of immortalized hepatocyte through transfer of oncogenic proteins and RNAs. Carcinogenesis 36, 1008-1018 (2015)

85. Ramakrishnaiah, $\mathrm{V}$. et al. Exosome-mediated transmission of hepatitis $\mathrm{C}$ virus between human hepatoma Huh7.5 cells. Proc. Natl. Acad. Sci. U. S. A. 110, 13109-13113 (2013).

86. Tucker, R. \& Pedro, A. Blood-derived non-extracellular vesicle proteins as potential biomarkers for the diagnosis of early ER + breast cancer and detection of lymph node involvement. F1000Res. 7, 283 (2018).

87. Griffiths, S. G., Cormier, M. T., Clayton, A. \& Doucette, A. A. Differential proteome analysis of extracellular vesicles from breast cancer cell lines by chaperone affinity enrichment. Proteomes 5, pii: E25 (2017).

88. Thomas, S. N. et al. Exosomal proteome profiling: a potential multi-marker cellular phenotyping tool to characterize hypoxia-induced radiation resistance in breast cancer. Proteomes 1, 87-108 (2013).

89. Moon, P. G. et al. Identification of developmental endothelial locus-1 on circulating extracellular vesicles as a novel biomarker for early breast cancer detection. Clin. Cancer Res. 22, 1757-1766 (2016).

90. Chow, A. et al. Macrophage immunomodulation by breast cancer-derived exosomes requires Toll-like receptor 2-mediated activation of NF-kappaB. Sci. Rep. 4, 5750 (2014)

91. Vardaki, I. et al. Periostin is identified as a putative metastatic marker in breast cancer-derived exosomes. Oncotarget 7, 74966-74978 (2016).

92. Kavanagh, E. L. et al. Protein and chemotherapy profiling of extracellular vesicles harvested from therapeutic induced senescent triple negative breast cancer cells. Oncogenesis 6, e388 (2017).

93. Gangoda, L. et al. proteomic profiling of exosomes secreted by breast cancer cells with varying metastatic potential. Proteomics 17, https:/doi.org/ 10.1002/pmic.201600370 (2017).

94. Domenyuk, V. et al. Plasma exosome profiling of cancer patients by a next generation systems biology approach. Sci. Rep. 7, 42741 (2017).

95. Sinha, A., Ignatchenko, V., Ignatchenko, A., Mejia-Guerrero, S. \& Kislinger, T. Indepth proteomic analyses of ovarian cancer cell line exosomes reveals differential enrichment of functional categories compared to the $\mathrm{NCl} 60$ proteome. Biochem. Biophys. Res. Commun. 445, 694-701 (2014).
96. Escrevente, C. et al. Sialoglycoproteins and N-glycans from secreted exosomes of ovarian carcinoma cells. PLoS ONE 8, e78631 (2013).

97. $\mathrm{Yi}, \mathrm{H}$. et al. Exosomes mediated pentose phosphate pathway in ovarian cancer metastasis: a proteomics analysis. Int. J. Clin. Exp. Pathol. 8, 15719-15728 (2015).

98. Yi, H. et al. High-grade ovarian cancer secreting effective exosomes in tumor angiogenesis. Int. J. Clin. Exp. Pathol. 8, 5062-5070 (2015).

99. Adamczyk, K. A. et al. Characterization of soluble and exosomal forms of the EGFR released from pancreatic cancer cells. Life. Sci. 89, 304-312 (2011).

100. Jin, $\mathrm{H}$. et al. Exosomal zinc transporter ZIP4 promotes cancer growth and is a novel diagnostic biomarker for pancreatic cancer. Cancer Sci. 109, 2946-2956 (2018).

101. Zheng, J. et al. Extracellular matrix proteins and carcinoembryonic antigenrelated cell adhesion molecules characterize pancreatic duct fluid exosomes in patients with pancreatic cancer. HPB 20, 597-604 (2018).

102. Blomme, A. et al. Myoferlin is a novel exosomal protein and functional regulator of cancer-derived exosomes. Oncotarget 7, 83669-83683 (2016).

103. Klein-Scory, S. et al. New insights in the composition of extracellular vesicles from pancreatic cancer cells: implications for biomarkers and functions. Proteome Sci. 12, 50 (2014).

104. Yu, Z. et al. Pancreatic cancer-derived exosomes promote tumor metastasis and liver pre-metastatic niche formation. Oncotarget 8, 63461-63483 (2017).

105. Castillo, J. et al. Surfaceome profiling enables isolation of cancer-specific exosomal cargo in liquid biopsies from pancreatic cancer patients. Ann. Oncol. 29, 223-229 (2018).

106. Shin, S. J. et al. Unexpected gain of function for the scaffolding protein plectin due to mislocalization in pancreatic cancer. Proc. Natl. Acad. Sci. USA 110, 19414-19419 (2013).

107. Chaiyadet, S. et al. Carcinogenic liver fluke secretes extracellular vesicles that promote cholangiocytes to adopt a tumorigenic phenotype. J. Infect. Dis. 212, 1636-1645 (2015).

108. Khoontawad, J. et al. Differential protein expression marks the transition from infection with opisthorchis viverrini to cholangiocarcinoma. Mol. Cell. Proteom. 16, 911-923 (2017).

109. Taverna, S. et al. Curcumin modulates chronic myelogenous leukemia exosomes composition and affects angiogenic phenotype via exosomal miR-21. Oncotarget 7, 30420-30439 (2016).

110. Paggetti, J. et al. Exosomes released by chronic lymphocytic leukemia cells induce the transition of stromal cells into cancer-associated fibroblasts. Blood 126, 1106-1117 (2015)

111. Huan, J. et al. Coordinate regulation of residual bone marrow function by paracrine trafficking of AML exosomes. Leukemia 29, 2285-2295 (2015).

112. de Andrade, A. et al. Extracellular vesicles from oral squamous carcinoma cells display pro- and anti-angiogenic properties. Oral. Dis. 24, 725-731 (2018).

113. Ono, K. et al. HSP-enriched properties of extracellular vesicles involve survival of metastatic oral cancer cells. J. Cell. Biochem. 119, 7350-7362 (2018). 